\title{
INCISIONAL ANEURYSMS OF THE LEFT VENTRICLE
}

\author{
BY \\ W. F. KERR, D. E. L. WILCKEN, AND R. E. STEINER \\ From the Departments of Surgery, Medicine, and Radiology, Postgraduate Medical School of London
}

Received June 18, 1960

In the treatment of stenosis of the valves of the heart, it is common surgical practice to pass an expanding dilator or valvulotome through the cavity of the ventricle to the affected valve. To permit insertion of the instrument an incision about $1 \mathrm{~cm}$. long is made near the apex of the ventricle, avoiding small coronary vessels, and the wound is repaired afterwards with two or three silk or linen sutures. The advantages and the immediate difficulties and dangers of such a procedure have been well documented and widely discussed, but latecomplications attributable to the ventriculotomy itself have attracted scant attention. We therefore report the occurrence of three left ventricular aneurysms following ventriculotomy and discuss their development, diagnosis, and treatment. Of these three incisional aneurysms, one was false and two were true.

\section{CASE REPORTS}

Case 1. A 50-year old Yugoslav man was admitted early in 1958 with severe aortic stenosis and mild aortic regurgitation. The first operation (12/2/58, Mr. W. P. Cleland) was a transventricular dilation of the heavily calcified aortic valve, using a 3-bladed dilator. The ventriculotomy was repaired with two silk sutures and the pericardium was sutured over the wound in the heart. Recovery from the operation was retarded by a staphylococcal pulmonary infection, the first signs of which appeared on the third day after operation. A course of erythromycin and novobiocin was started. The following day the clinical and radiological appearances suggested a collection of fluid at the left lung base, but aspiration yielded only a small quantity of turbid yellow fluid which on culture produced a heavy growth of Staphylococcus pyogenes, sensitive to erythromycin and novobiocin. His temperature settled to normal within the next few days and thereafter progress was satisfactory.

The assessment of his condition on discharge from hospital was that there had been good relief of the stenosis at the expense of some increase in regurgitation. Re-expansion of the left lower lobe was incomplete and there was a good deal of residual thickening of the pleura.

On $27 / 3 / 58$, i.e. two weeks after his discharge, and six after the operation, he was readmitted. He had been well until three days earlier, when he began to shiver and feel ill, with pains all over his body. There had been anorexia and vomiting, but no cough and no sputum. A few hours before readmission he had begun to suffer pain in the anterior end of his scar where previously there had been only mild discomfort. His temperature was $100 \cdot 4^{\circ} \mathrm{F}$. Except for tachycardia and a small rise in jugular venous pressure the physical signs were the same as when he left hospital and the $\mathrm{X}$-ray appearances (Fig. 1) were not materially changed. Hæmoglobin had dropped to $10.6 \mathrm{~g}$. per $100 \mathrm{ml}$. and the white cell count was 9000 per c.mm., with a relative neutrophil leucocytosis. There was no obvious cause for his illness.

After blood cultures had been set up, he was given tetracycline and kept under observation. His temperature rose during the night to $102^{\circ} \mathrm{F}$, and the pain in his chest increased, but there were no new signs until the following night, when a tender swelling appeared under the anterior end of the scar. By morning it was about $10 \mathrm{~cm}$. in diameter, and $3-5 \mathrm{~cm}$. in depth. The skin covering it 


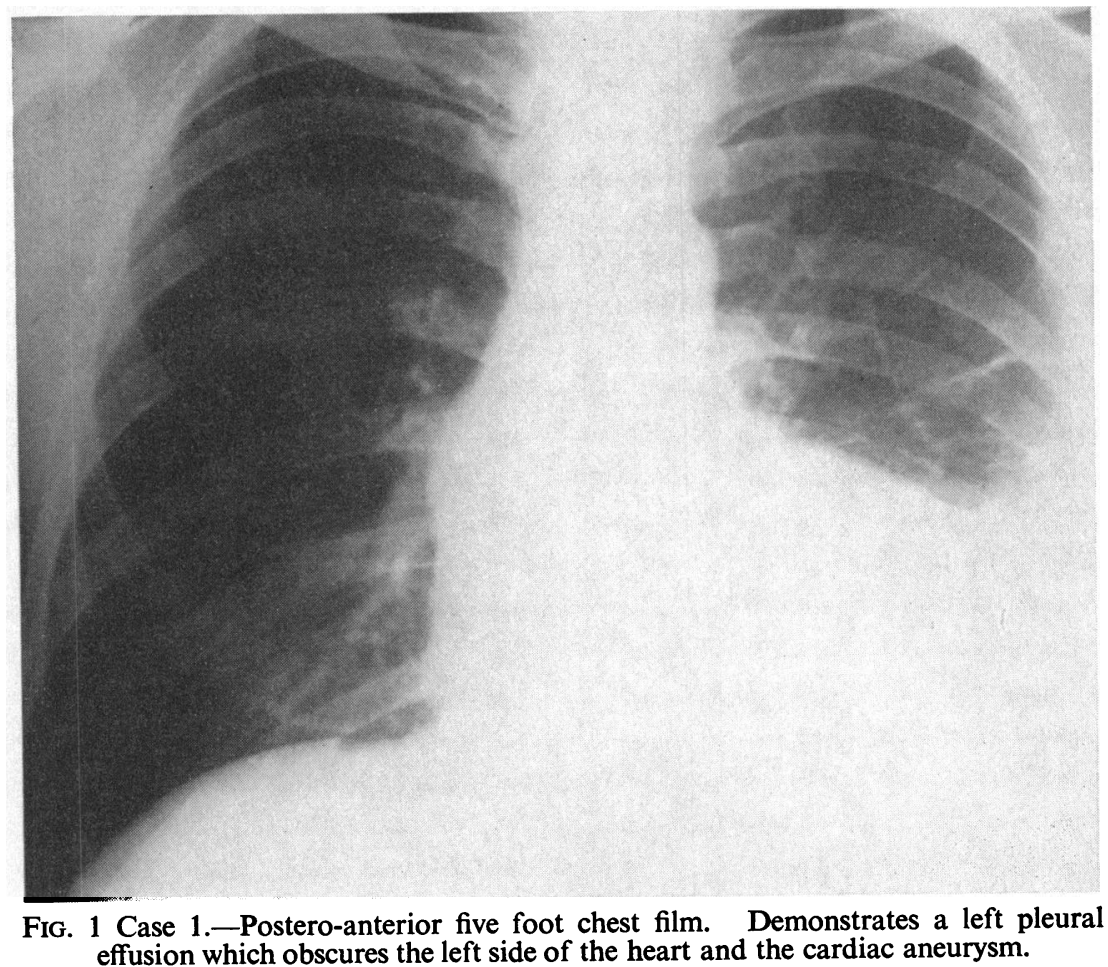

was slightly reddened and tense and pressure caused pitting. The whole area pulsated. It was thought to represent empyema necessitatis with transmitted pulsation from the greatly enlarged ventricle beneath it. Aspiration of the swelling itself yielded only a small quantity of dark blood, and aspiration of the pleural sac failed to locate pus.

Second operation $(29 / 3 / 58)$ (W. F. K.). Under local anæsthesia a 3-inch incision was made through the anterior end of the old scar, where the swelling was greatest. The tissues looked fresh and uninfected. In the intercostal space, the pleura covered a mass of clotted blood which seemed only a few days old. Suddenly from the depths of the wound there came a gush of bright red blood, obviously from the heart. Temporary control was achieved with a gauze pack and pressure, while blood transfusion was started and general anæsthesia induced. The pack was therefore sewn into the wound, and a firm dressing applied. There was no evidence of further bleeding until the following morning when discoloration and induration of the left flank were noted.

Third operation (30/3/58, Mr. W. P. Cleland). With 16 pints of blood cross-matched, the left femoral artery was exposed and a large cannula tied into it for rapid arterial transfusion. The patient was turned into the right lateral position and the left side of the chest was entered by resecting the posterior half of the 6th rib. As expected, the lung was firmly adherent to the chest wall, and mobilization was not easy. The incision was cautiously carried forward, removing short sections of rib, and mobilizing the lung until the region of the apex of the heart was reached. There a fairly large false aneurysm was found, confined mainly by pericardium. Part of the wall was thin and friable, and this was where leakage had occurred. Hæmorrhage was profuse when the plug inserted the previous day was displaced, but a gauze swab and digital pressure controlled it while the remainder of the rib was removed, and lung, diaphragm, and pericardium were separated from the heart. When the apex of the heart had been fully exposed, blood could be seen spurting through the ventriculotomy, which showed little evidence of healing. The two threads with which it had been sutured lay loosely across it; they were picked out and preserved for bacteriological examination. 
The myocardium was resutured with deeply-placed mattress stitches of chromic catgut, and reinforced with the wall of the aneurysm. Blood lost during the operation measured $3000 \mathrm{ml}$; ; arterial transfusion was not required.

Recovery was slow at first, and was further retarded by a right-sided pulmonary infarct. Subsequent progress was rapid and the patient left for Yugoslavia on 3/6/58. Staph. albus was cultured from the cardiac sutures (the same organism was found in the blood cultures), and a buried nylon suture from the chest wall grew Staph. pyogenes.

Case 2. A woman of 53 years, whose only valvular lesion was severe mitral stenosis, suffered from

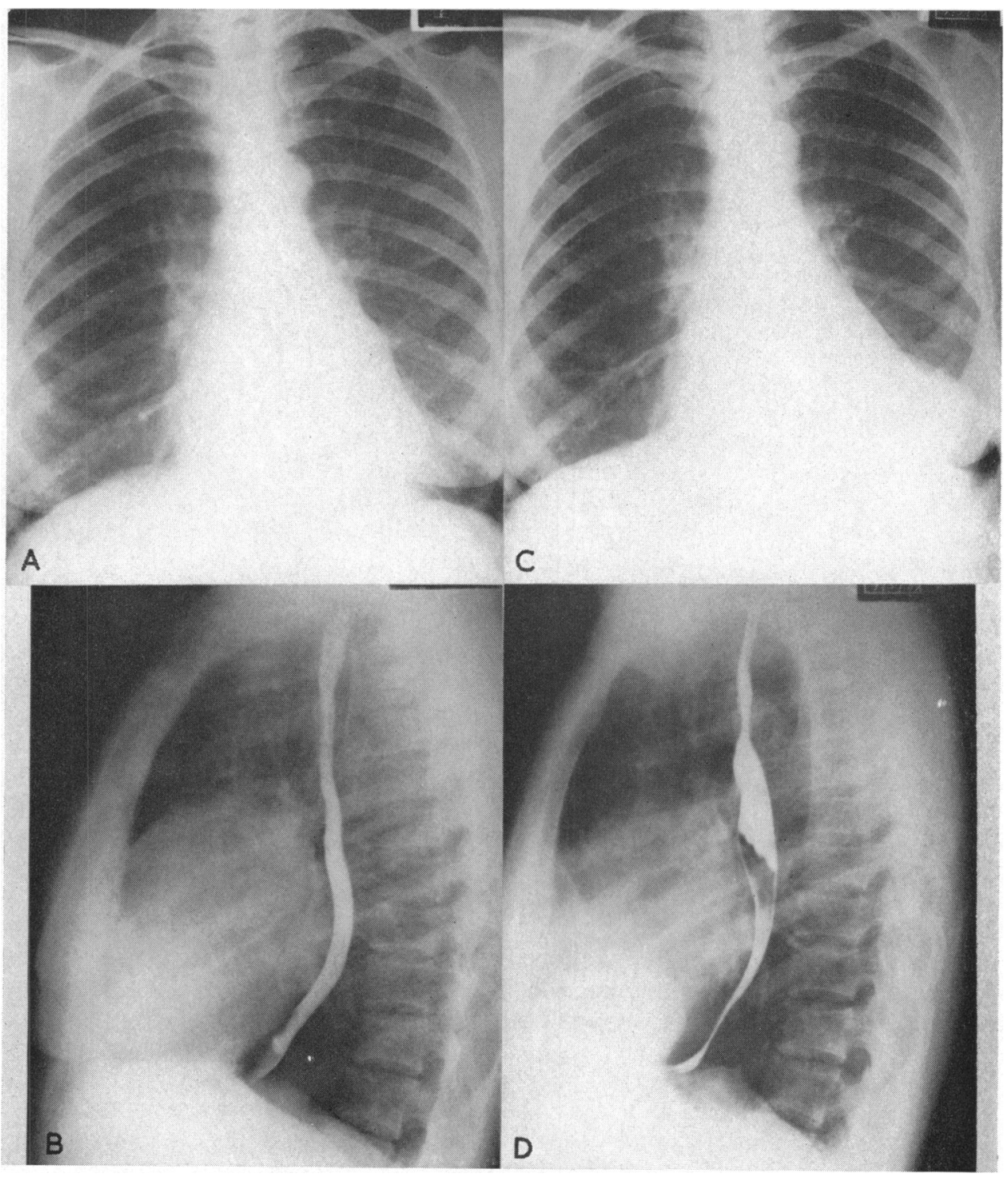

Fig. 2, Case 2.- $A$ and $B$. Pre-operative films, postero-anterior and lateral, demonstrate the enlarged left atrium. $C$. Postero-anterior five foot chest film demonstrates a bulge along the left cardiac border continuous with the apex of the heart. $D$. Lateral film demonstrates an oval shadow superimposed on the heart just above the diaphragm. 
mild chest pain on exertion in addition to the usual symptoms of mitral obstruction. After exertion there was S-T segment depression of $2 \mathrm{~mm}$. in lead V7-a positive electrocardiographic effort test. At the first operation $(31 / 10 / 58$, W. F. K.) Tubbs' transventricular dilator was used and a fullsized mitral orifice was obtained without the production of palpable regurgitation. The incision in the ventricle was repaired with three linen thread sutures and the pericardium was reconstituted over it.

In the early postoperative period low-grade pyrexia was attributed to a persistent small pleural effusion, which was still present when she was discharged four weeks after the operation. When she came to the outpatient clinic six weeks later she was found to be in mild congestive failure. By that time a pansystolic murmur was audible at the apex, and mitral regurgitation, together with inadequately controlled atrial fibrillation, was thought to be responsible. At her routine visit six months after operation she reported that she was much more active than before. Her only complaint was of an "expanding sensation" in the chest which did not distress her. The signs of failure were no longer present; the left ventricular impulse was rather diffuse; the apical systolic murmur was
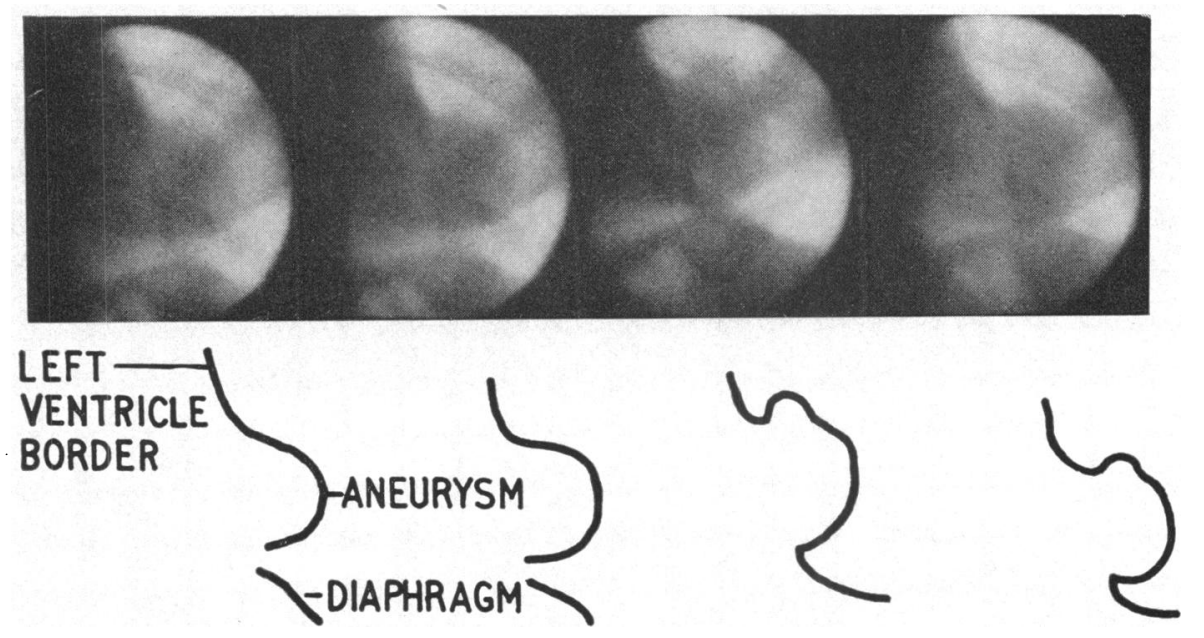

FIG. 3, Case 2.-Cine radiography of the aneurysm at 32 films a second; every 8 th film is printed to demonstrate the variation in size of the aneurysm.

unchanged; a barely audible short mid-diastolic murmur had appeared. Her electrocardiogram was identical with the one before operation but the chest X-ray, the first taken since her discharge, was strikingly altered. On the postero-anterior view, there was a large rounded bulge continuous with the left ventricular shadow at the apex (Fig. 2). Paradoxical pulsation was observed on fluoroscopy and recorded by cineradiography (Fig. 3) and an angiocardiogram showed contrast medium flowing freely from the left ventricle into the bulge (Fig. 4). The diagnosis of left ventricular aneurysm was incontrovertible.

Second operation (22/5/59, Mr. W. P. Cleland). With equipment for left ventricular by-pass in readiness, the chest was opened by resecting the whole of the 6th rib. There were a few pleural adhesions, and the aneurysm was immediately apparent (Fig. 5). The neck of the sac was isolated without disturbing the fundus, by undermining the pericardium adherent to the surrounding ventricle. With a Crafoord clamp positioned to control the neck of the aneurysm, the sac was cleared of pericardium without endangering the phrenic nerve.

After full exposure the aneurysm was seen to have a thin wall and a narrow neck, the external dimensions of the latter being $3 \mathrm{~cm}$., in the direction apex-to-base, by $1 \mathrm{~cm}$. The neck was clamped and permanently occluded with four horizontal mattress sutures of strong chromic catgut passed through the fibro-muscular ring deep to the clamp. On opening the sac after removal of the clamp 


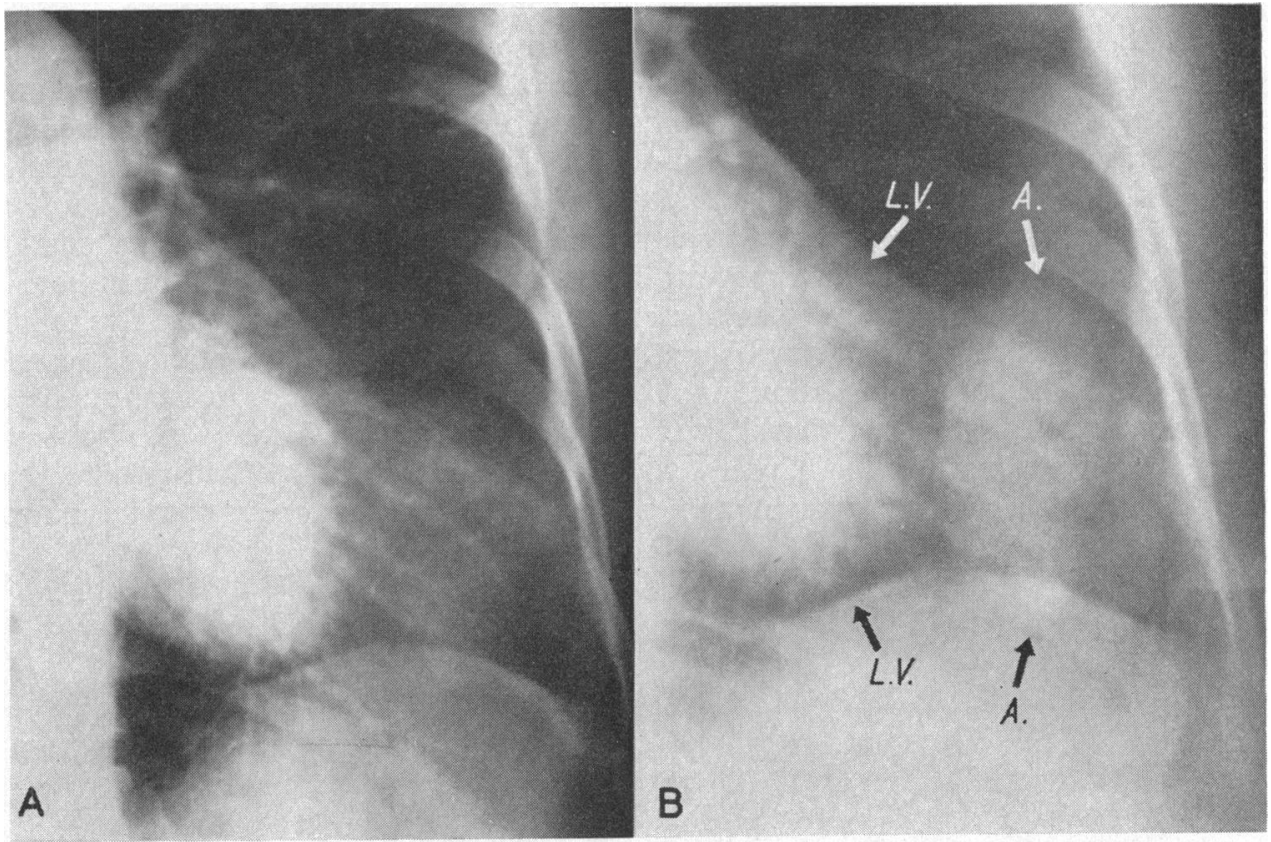

FIG. 4, Case 2.- $A$ and $B$. Two films from the angiocardiographic series to demonstrate filling of the aneurysm from left ventricle. Note the delayed filling of the aneurysmal sac in $(B) . \quad L . V .=$ left ventricle. $\mathrm{A}=$ aneurysm.

a small clot was seen, looking so fresh that it was thought to have formed only after closure of the neck. The internal surface of the neck was smooth, white, and glistening (Fig. 5b), and the three linen threads used for repairing the original ventriculotomy were found close together in the fundus - proving that the aneurysm was a true one. There were no signs of old or recent infection. The redundant portion of the sac was cut away, and the cuff that remained was sutured with an everting layer of silk mattress stitches and another layer of continuous silk. The whole area was covered with pericardium and the chest closed. The blood loss measured $400 \mathrm{ml}$.

Recovery was uneventful. The patient was allowed up after two weeks and discharged to a convalescent home after three. Six months after the operation she was very well. There was a normal left ventricular impulse and no systolic murmur. On the chest X-ray there were signs of pleural thickening in the costo-phrenic angle but the outline of the left ventricle was normal. The electrocardiogram was unchanged.

Dr. I. Doniach's report on the histology of the aneurysmal sac follows. "... Mainly fibrous tissue with thrombus adherent to the inner surface and beginning to organize. At one point in the wall there is a localized collection of plasma cells, lymphocytes, macrophages and foreign-body giant cells, containing suture material. No heart muscle is recognizable. The collagen is well vascularized, and shows increasing differentiation from within outwards. There are pieces of pericardium stuck to its outer surface ..." (Fig. 5c, d).

Case 3. A man aged 26, with severe aortic stenosis and minimal aortic regurgitation, was operated upon by Mr. W. P. Cleland on 30/3/55. A satisfactory reduction in the aortic gradient was achieved by transventricular dilatation of the valve. During cardiac manipulations the patient became cyanosed, and took some time to recover. By the following day there were electrocardiographic signs of an anterior myocardial infarct consisting of elevation of S-T segments in leads 1 , V2, and V3, with reciprocal depression in V4R and V1. He was kept in bed for three weeks, and discharged to a convalescent home after four. Ten weeks after operation the $T$ waves were inverted 


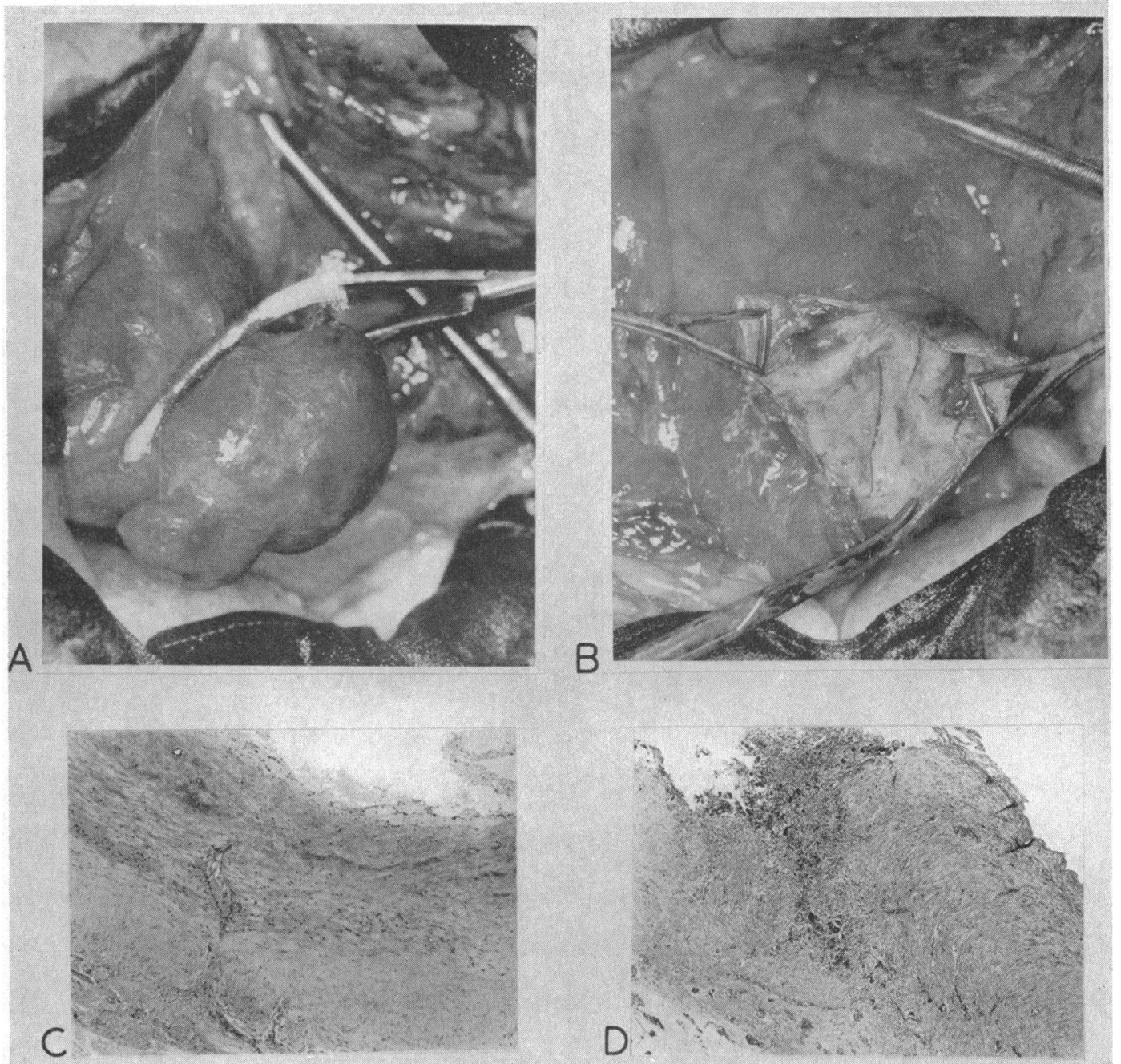

Fig. 5, Case 2. $-A$ and $B$. Aneurysm exposed at operation and then opened. $C$. Wall of true aneurysm. The endocardial surface is uppermost. Three layers can be seen. On the endocardial surface is organizing thrombus. Below this, and staining more darkly is a layer of young collagen formed from organized thrombus. The zone nearest the epicardial surface consists of older dense fibrous tissue. H. \& E.: $\times 60$. $D$. Wall of true aneurysm. The endocardial surface lies uppermost and to the right. Section cut from near a stitch inserted to close the ventriculotomy wound at the time of the mitral valvotomy and now lying in the wall of the aneurysm. Marked foreign body reaction with giant cells can be seen. H. \& E.: $\times 38$.

in leads 1, 2, and V2 to V7; they gradually became upright again during the next two months (Fig. 6). A chest film taken 11 weeks after the operation revealed a protrusion continuous with the left cardiac border which had not been present six weeks earlier (Fig. 7).

There was no alteration in the size of the protrusion radiologically over the next $4 \frac{1}{2}$ years and no action was taken. Experience with the two cases already described, suggested his readmission in October 1959. He presented then the signs of moderate aortic stenosis without regurgitation. There was no clinical evidence of a cardiac aneurysm. On the electrocardiogram some flattening of the $T$ waves in the left ventricular leads had appeared in the last two years, but there were no other abnormalities. Paradoxical pulsation could be seen on fluoroscopy, and an angiocardiogram confirmed that it was an aneurysm. 


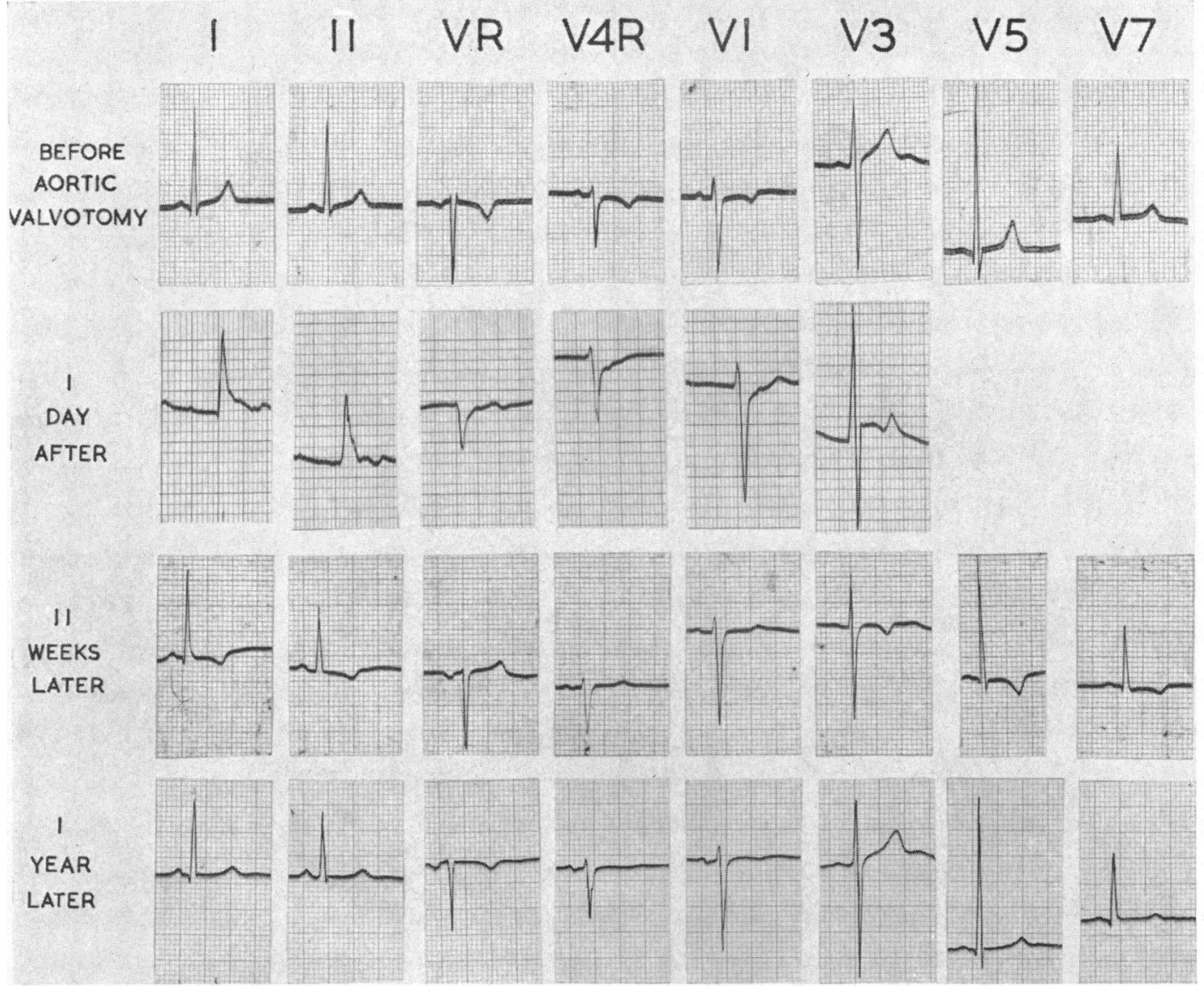

Fig. 6.-Electrocardiograms of Case 3 show the gradual resolution of the infarct pattern seen the day following operation.

Second operation (10/11/59, Mr. H. H. Bentall). The residual aortic stenosis was not severe enough to warrant a second valvotomy and only a repair of the aneurysm was planned. The chest was opened by an anterolateral incision in the 6th intercostal space; the lung was totally adherent. When the phrenic nerve had been dissected out and retracted, the pericardium was incised round the base of the aneurysm and mobilized from the heart, leaving undisturbed the investment of the fundus of the sac, and the aneurysm was revealed as a small tense hemisphere with obvious paradoxical pulsation. The base was $2.5 \mathrm{~cm}$. across, and this appeared to be the size of the defect in the heart muscle, the "neck" therefore being large in relation to the size of the aneurysm. Palpation was not attempted for fear of dislodging emboli. The shape of the aneurysm was such that a clamp could not easily be placed across its base, but the neck was securely closed by passing two inter-locking mattress sutures of strong catgut under the aneurysm. As in the previous case, the lining of the sac was smooth and glistening, there was no preformed clot, and after resection one of the silk stitches inserted at the original operation was found in the portion removed. It was therefore a true aneurysm. The fibrous rim of the neck was sutured with continuous silk and several interrupted silk sutures. There was insufficient pericardium to cover the repair.

The patient made a good recovery from the operation and was discharged to a convalescent home in four weeks.

Dr. I. Doniach reported on the histology of the aneurysm wall as follows. "... shows a thin 


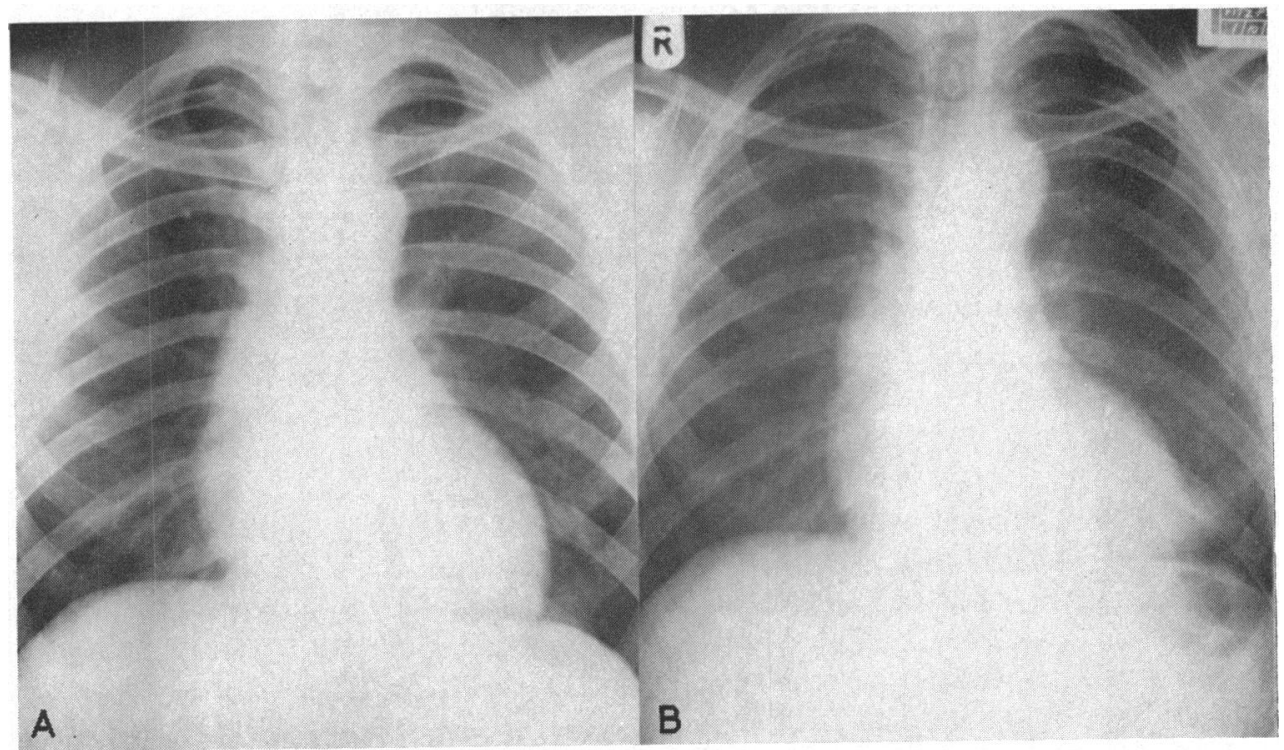

FIG. 7, Case 3.- $A$. Pre-operative five-foot postero-anterior chest film demonstrates enlargement of the left ventricle with a smooth left cardiac border. $B$. Post-operative five-foot postero-anterior chest film demonstrates a bulge continuous with the apex of the heart just above the diaphragm.

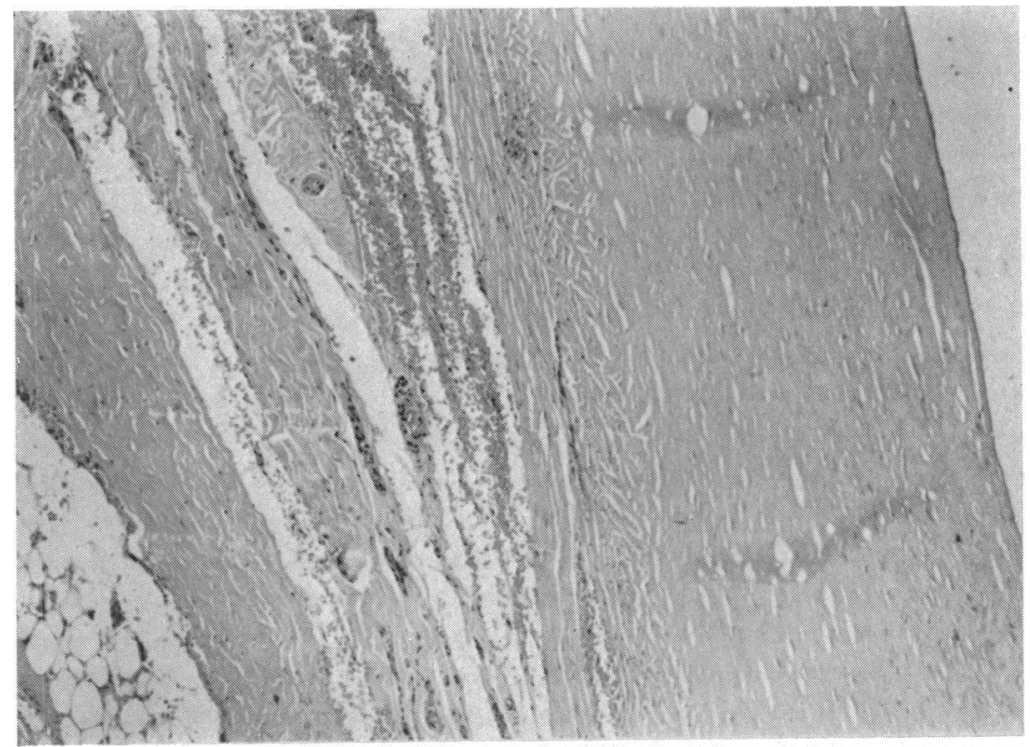

FIG. 8, Case 3.-Wall of true aneurysm. The endocardial surface lies to the right of the section. The wall consists of dense fibrous tissue more mature than that seen in Case 2. The aneurysm had been present for $4 \frac{1}{2}$ years, while that in Case 2 had been present for only 6 months. H. \& E.: $\times 70$.

collagenous wall with one deposit of calcium. The endocardial endothelium is present, but there is no muscle and almost no elastic tissue. There are several small vessels in ine outer area-one artery shows endarteritis obliterans ..." (Fig. 8). 


\section{Discussion}

Types of Aneurysms. Aneurysms of the heart, like aneurysms of arteries, may be true or false. A true aneurysm is part of the heart (Fig. 9). A saccular aneurysm with a narrow neck develops from a small area of weakness in the myocardium. If the area affected is larger, a wide-necked sac may develop, or there may be a diffuse bulge of the wall of the heart. Sooner or later all muscle in the wall of the sac is replaced by fibrous tissue.

A false aneurysm, on the other hand, lies outside the heart, and communicates with it through a perforation in the myocardium. Such an aneurysm develops when blood, leaking persistently from the ventricle, is contained by the surrounding structures (Fig. 9). Adhesions at first are fibrinous; later they become organized, and form a tough wall of fibrous tissue indistinguishable even on microscopic examination from the wall of a chronic true aneurysm.

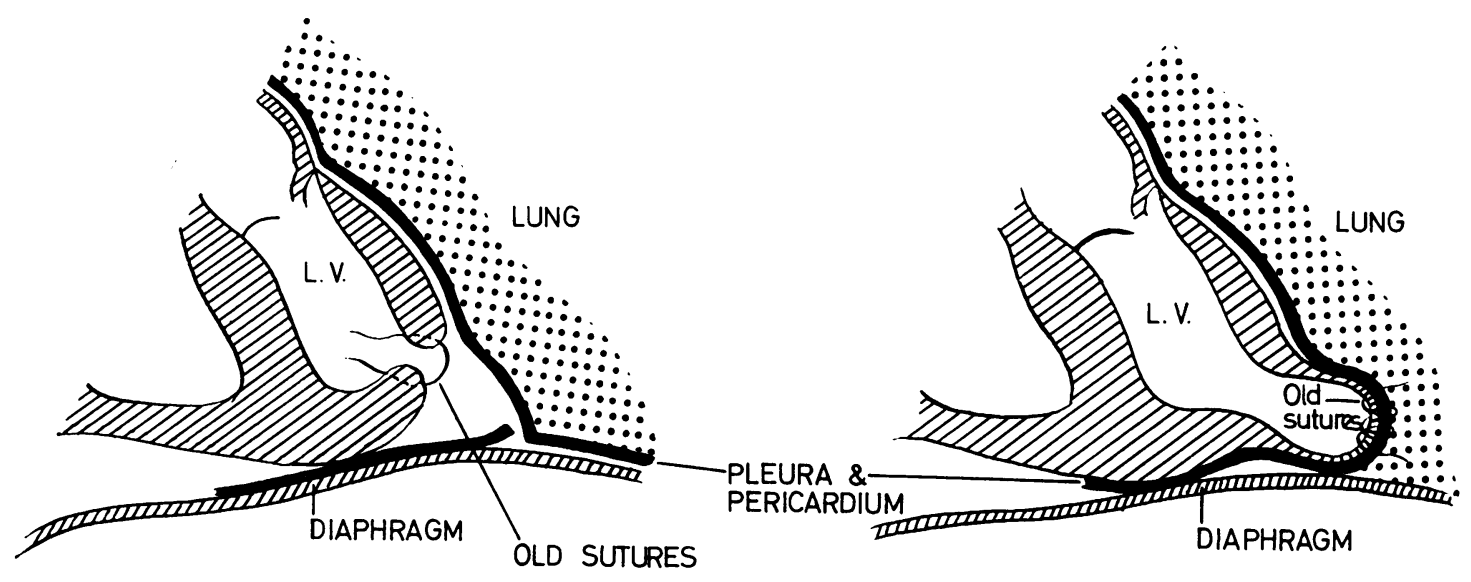

FALSE

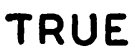

FIG. 9.-The position of old suture material indicates whether the aneurysm is true or false.

Incidence of Traumatic Aneurysms. Aneurysms of the heart that are the result of infarction and myomalacia are not uncommon, but aneurysms resulting from trauma (and this includes surgical incisions) are extremely rare if the scarcity of reported cases is any guide. They have been noted after non-penetrating injuries (Bright and Beck, 1935; Barber, 1944), but the interval between the injury and the opportunity for pathological study obscures the details of pathogenesis. Most articles about penetrating wounds are concerned with the recognition and treatment of acute cardiac tamponade, and few give late results. Even in times of war, cardiac aneurysms seem to have occasioned little interest or concern, and there is no mention of them in Harken's (1946) report on the late removal of 56 missiles from the heart and its environs, although it is stated that the only fragment that involved the left ventricle was found in a "small cystic myocardial hernia".

An interesting left ventricular aneurysm was reported by Lyons and Perkins (1958). It was partially calcified when its presence was first noted on an X-ray taken 12 years after suture of a stab wound which had penetrated the left ventricle. Two cerebral emboli provided the main indication for resection, successfully undertaken 21 years after the original assault. Another left ventricular aneurysm was the result of a surgical accident; in trying to split the lateral commissure of the mitral valve the surgeon's finger slipped and perforated the ventricle from within. The laceration was immediately repaired, but an aneurysm developed at the site within three months. Smith et al. (1957) have described how it was diagnosed and repaired. 
A few aneurysms have occurred after deliberate surgical incisions. We have seen one in a boy aged 13 with Fallot's tetralogy: after a closed pulmonary valvotomy five years previously, he came to operation for complete correction on 26/6/59, and when the heart was exposed the true aneurysm was an unexpected finding. We are indebted to Mr. L. Fatti of Johannesburg (1959) for particulars of a second case, a young woman with Fallot's tetralogy who, in 1951, had a pulmonary valvotomy at which Brock's valvulotome and fenestrated dilator was used; and eighteen months later she was operated on again for an aneurysm which had developed at the site of the ventricular incision: this was a true aneurysm, with a circular neck about $3 \mathrm{~cm}$. in diameter. A somewhat similar example was reported by Campbell et al. (1954): four months after closed pulmonary valvotomy for Fallot's tetralogy, post-mortem examination disclosed a large aneurysm at the site of the cardiotomy. A post-operative aneurysm of the right ventricle, though not an incisional one, was described by McCord and Blount (1955): seven weeks after infundibular resection in a case of Fallot's tetralogy a pulsating swelling was noted in the præcordium and, at the subsequent operation, a large thin-walled true aneurysm of the infundibular chamber was found. Derra and Loogen (1959) also reported a case in which a true incisional aneurysm of the right ventricle "the size of an apple" was resected: this was six years after a pulmonary valvotomy by Brock's closed method.

Aneurysms after ventriculotomy are equally uncommon on the left side of the heart. Segal et al. (1957) reported two after aortic valvotomy, diagnosed by X-ray and electrocardiography; there is no information as to type. The only other example we have been able to find is one reported by Smith et al. (1957, Case 2), a false aneurysm in a 38-year-old woman who had had aortic stenosis relieved by the transventricular route, at the same time as mitral stenosis was treated by the left atrial route: an abnormality visible on X-rays taken early in the post-operative period was recognized as an aneurysm 18 months later and successfully dealt with. In another case an "aneurysmal dilatation" was discovered at necropsy on a woman who died ten months after partial valvulotomy for mitral stenosis (Smithy et al. 1950); where the valvulotome had been inserted at the apex of the ventricle there was attentuation of the myocardium over an area $1.7 \mathrm{~cm}$. in diameter, and the thickness at the centre was reduced to $3 \mathrm{~mm}$.: this appears to have been an early stage of a true aneurysm.

Healing of Wounds of the Heart. Wounds of the myocardium, like wounds of other specialized tissues lacking regenerative power, heal by fibrous union with the formation of a cicatrix. The process was studied in the left ventricles of dogs by Thomas and Ziffren (1952), and their findings, summarized below, were confirmed by Warren et al. (1958). A constant feature of wound closure was a zone of necrosis within the suture line, and in the cases of wound rupture it was found that the sutures had produced considerable necrosis. In those wounds that did not break down granulation tissue rapidly invaded the necrotic muscle, completely replacing it in two or three weeks to produce a scar that at first was as thick as the wall of the ventricle. When the length of the incision was limited to $2 \mathrm{~cm}$. attenuation of the scar did not occur to any great extent, but with wounds 3-4 $\mathrm{cm}$. long, which did not divide any visible blood vessels, broadening and thinning of the scar began within a month and progressed until the scar measured half or less of its original thickness. This process was most striking near the apex. In the healing of large incisions in the right ventricle Warren et al. (1957) were impressed by the areas of myocardial thinning that constantly occurred between the wound and the septum when the incision was made without regard to the branches of the right coronary artery. (In the dog, this artery supplies most of the right ventricle.) No aneurysms occurred during these experiments. Beck (1944) recounts how he tried, and failed, to produce aneurysms by localized crushing of the myocardium, and by ligation of a coronary artery.

In man short incisions for the insertion of valvulotomes are made at sites selected to avoid blood vessels, and are carefully sutured afterwards. It is not surprising therefore that they nearly always heal well, and seldom give rise to trouble.

\section{ATiology}

False Aneurysms. In Case 1 the aneurysm was patently the result of staphylococcal infection of the suture line. The acute pulmonary and pleural reaction that developed soon after the operation 
points to the respiratory tract as the portal of entry, and the spread to the heart could have been direct or blood-borne. It seems probable that wound disruption occurred in the sixth week, when the acute symptoms developed, and the thin and friable appearance of the sac walls at the reparative operation supported this view.

The two aneurysms reported by Smith et al. (1957) were false and so, apparently, was that of Lyons and Perkins (1958): these were attributed by the authors not to infection, but to faults in the technique of suturing. Bailey et al. (1958), who regard most if not all traumatic aneurysms as false, believe that the fault lies in failure to place the sutures through the full thickness of the muscle, thereby facilitating dissection. Thomas and Ziffren (1952) and Warren et al. (1958) conclude from their experiments that the best results are obtained by maximum apposition of the incised surfaces. There is no doubt that in suturing the ventricle this should be the aim; but failure to take the full thickness of the muscle is not entirely satisfying as an explanation of the disruption that gives rise to a false aneurysm. In other situations, such as in bronchial closure or intestinal anastomosis, ischæmic or infective necrosis is largely responsible for wounds giving way, and the same pathological sequence occurs in the heart. One stitch badly placed or tied too tightly may cause excessive necrosis and subsequent disruption of part of the wound. The break-down is an early occurrence, but the aneurysm to which it gives rise may not be discovered until much later.

True Aneurysms. Reference has already been made to the presumption that traumatic aneurysms are characteristically false, an opinion that cannot go unchallenged in the light of our experience and that of Smithy et al. (1950). The aneurysms associated with coronary artery disease are sequels of infarction (Parkinson et al., 1938; Schlichter et al., 1954) and we would suggest that infarction is also the antecedent of the traumatic true aneurysms, for in damaged hearts, interstitial hæmorrhage and œdema following instrumentation may devitalize surrounding muscle fibres to produce a small area of infarction in the heart wall.

We have seen one example of a proved infarct probably caused in this way. A woman fifty years of age underwent transventricular aortic valvotomy for severe calcific aortic stenosis, and died suddenly 12 days later. Autopsy showed the wound fairly well healed, and adjacent to it, although apparently not in direct continuity with it, an infarct measuring $25 \mathrm{~mm}$. in diameter which involved the full thickness of the ventricular wall and the papillary muscle. The diffuse infiltration with mononuclear and plasma cells indicated that its age was comparable with that of the incision (Fig. 10). There was no coronary artery occlusion to account for the infarct. Segal et al. (1957) had a similar experience: an aortic valvotomy patient died suddenly two weeks after operation and necropsy revealed an infarcted area of myocardium around the cardiotomy, and no evidence of occlusion of the coronary vessels.

They also mention another patient whose electrocardiogram immediately after transventricular aortic valvotomy showed the pattern of a large anterolateral infarct. Finally there is our third patient, who not only had electrocardiographic signs of an anterior infarct recorded on the first post-operative day (Fig. 6), but did subsequently develop a true aneurysm at the site of his cardiotomy. All these infarcts might be dismissed as embolic or coincidental but their close spatial and temporal relationships to ventricular trauma argues in favour of this as the proximate cause.

The condition of the heart before interference is of some importance as a predisposing cause. In aortic stenosis the muscle of the obstructed ventricle is notoriously unsound, often heavily infiltrated with fibrous tissue. In the patient reported by Smithy et al. (1950) with mitral valve disease, who developed aneurysmal dilation, it was noted at operation that the ventricle had a "yellowish mottled appearance suggesting extensive damage". The myocardium may have been previously damaged in our patient with mitral stenosis, for she had angina pectoris, and a positive electrocardiographic effort test.

The intraventricular pressure is another factor to be considered in the production of both true and false aneurysms. On theoretical grounds a high pressure should favour the development of an aneurysm, and it is interesting to note that of the five right ventricular aneurysms reviewed above, four occurred in cases of Fallot's tetralogy, in which the right ventricle usually operates at a raised pressure even after relief of the pulmonary stenosis, if the ventricular septal defect is not closed at the same time. In the fifth case (Derra and Loogen, 1959), the right ventricular pressure remained high after pulmonary valvotomy as was shown at repeat catheterization. In both of our cases of aortic stenosis the final left ventricular pressures were 


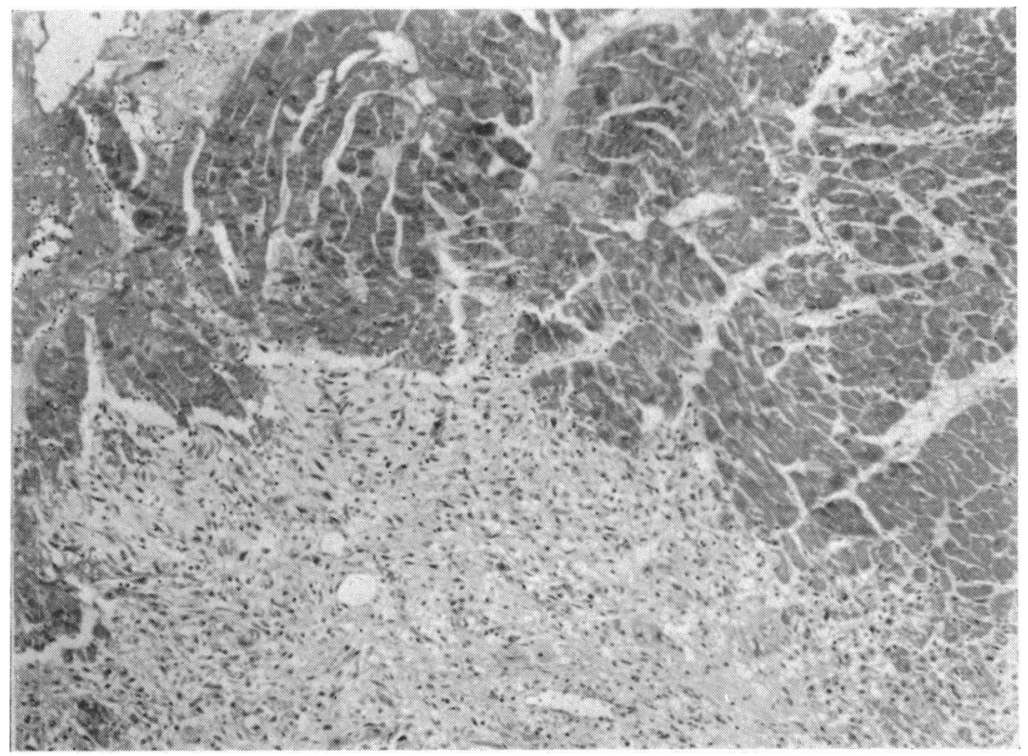

FIG. 10. Case F.K.-Section through the myocardial infarct showing necrotic heart muscle with young fibrous tissue infiltrated with mononuclear cells. H. \& E.: $\times 70$.

higher than normal, but in the patient operated on for mitral stenosis there was presumably no raised intr aventricular pressure to assist the development of the aneurysm.

The rate of growth of a cardiac aneurysm seems to be variable. A few cases of post-infarction aneurysm observed for from one to four years grew very slowly if at all (Parkinson et al., 1938). Smith et al. (1957) watched their two traumatic aneurysms increase in size within months, but that of Lyons and Perkins (1958) showed little alteration in nine years. The aneurysm in Case 3 had not appeared six weeks after operation, but by the 11th week it had reached the size at which it remained for the next $4 \frac{1}{2}$ years. The impression is gained that traumatic aneurysms develop fairly quickly in the train of the injury, although subsequent enlargement may be slow.

\section{DiAgNOSIS}

Clinical. In many cases a cardiac aneurysm produces no physical signs and in the remainder the signs are rarely diagnostic (Parkinson et al., 1938; Schlichter et al., 1954). Our third patient is an example of one with no clinical features to suggest the diagnosis. In our second, the rather diffuse apical pulsation and the apical systolic murmur were in retrospect undoubtedly produced by the aneurysm. In the first patient it seemed reasonable to suppose that empyema of the early postoperative period has recurred and broken through the chest wall to produce the præcordial swelling: the pulsation was thought to be transmitted.

Electrocardiographic. At the time the aneurysms developed there were no significant alterations in the electrocardiograms of the first two patients, although left ventricular hypertrophy in the first and digitalis in the second may have obscured $T$ wave changes. In the third patient, with the resolution of the signs of infarction, the $T$ waves over the left ventricle became upright again, and flattened only just before the second operation. This may be interpreted as an increase in left ventricular hypertrophy or as myocardial damage associated with the aneurysm. McCord and Blount (1955) record convincing $\mathrm{T}$ wave inversion in leads taken over the aneurysm in the case they described, and in the two cases of Smith et al. (1957) there were $T$ wave changes that may have been due to myocardial damage. Diminution in the amplitude of the $R$ waves or persistent $S-T$ segment elevation did not occur in our patients or in any similar published case. This is in sharp contrast to the pattern seen in the chronic aneurysms due to coronary artery disease, where these changes in leads taken over the 
aneurysm are constant features, and a dominant $\mathrm{R}$ wave in lead VR is not uncommon. Myers et al. (1948) attribute the $R$ wave changes to an absence of modifying potential from the electrically inactive tissue overlying the neck of the aneurysm and the surrounding relatively inactive fibrotic ventricular muscle: they suggest that hypertrophy of the opposite wall produces, within the ventricular cavity, electrical changes that are recorded unaltered through the electrically inactive area as S-T segment elevation. In traumatic aneurysms of the type described the neck is comparatively narrow, and we assume that adjacent relatively healthy muscle modifies cavity potential thus preventing S-T segment elevation and loss of $R$ wave amplitude.

The absence of the "characteristic" cardiographic changes therefore does not exclude a diagnosis of aneurysm: their absence in the presence of an aneurysm may be a favourable sign when surgery is being considered.

Radiological. Radiology provides the only reliable means of diagnosing cardiac aneurysms and specialized techniques are frequently necessary. Accuracy of diagnosis is particularly desirable if surgical intervention is a possibility.

A left ventricular aneurysm, true or false, appears on an X-ray film as a well circumscribed projection beyond the normal cardiac outline, seen best on a postero-anterior or slightly oblique view (Fig. 2c, 7b). In the lateral it appears as a separate rounded or oval shadow, partly or wholly superimposed on that of the heart (Fig. 2d). These appearances are visible only if the cardiac shadow is clearly defined and unobscured by pleural effusion, basal pleural thickening, or elevation of the diaphragm, conditions that are only too common after thoracic operations. A radiological diagnosis of aneurysm was therefore not possible in Case 1 (Fig. 1). Tomography might have helped to distinguish the heart shadow from the other structures and pleural fluid, but the only indication of anything unusual was the persistence of the "effusion", and this was consistent with the clinical diagnosis of empyema. The need for tomography becomes apparent when an abnormality is partially obscured by the overlying structures. In Case 2 the early stages of the aneurysm may have been concealed by the pleural effusion which did not clear until the patient had been discharged from hospital; the aneurysm showed plainly on the next film, taken five months later. In Case 3 the radiological abnormality was first noted eleven weeks after operation, and it remained unchanged for four and a half years.

Aneurysm is suggested but not proven by the appearances described. In the differential diagnosis encysted pleuro-pericardial effusions, localized thickening of the pleura, intrapleural fibrin bodies, and organized intrapericardial blood-clot must be considered and excluded. Gradual increase in size strengthens a suspicion of aneurysm, but failure to do so does not exclude this diagnosis, e.g. Case 3. Systolic expansion observed on fluoroscopy, or on the permanent record obtained by cineradiography, is good evidence in favour of aneurysm (Fig. 3) but it will not be seen if the sac is thrombosed. Angiocardiography is the most convincing of the specialized techniques, for if contrast medium is demonstrated entering the bulge from the left ventricle, the diagnosis of aneurysm is established. True and false sacs cannot be distinguished, but the procedure may yield information helpful to the surgeon by showing the size of the neck and suggesting the presence or absence of clot seen as a filling defect. On the other hand where the sac is full of thrombus or the neck is very narrow insufficient contrast material may enter the aneurysm to show radiologically.

Certain points may be mentioned in connection with the technique of angiocardiography. In our cases rapid right atrial injection, with the aid of a Talley pump, of $60 \mathrm{ml}$. of 85 per cent hypaque through a large cardiac catheter (National Institute of Health cardiac catheter no. 9 blocked end side hole) demonstrated the left ventricular aneurysms quite satisfactorily. It is important to realize that opacification of the aneurysmal sac may be delayed, and late films should be exposed. Contrast material showed in its greatest concentration in the aneurysms after the bulk of the injection had passed through into the general circulation (Fig. 4).

Other routes of injection have been recommended. Smith et al. (1957) injected into the left atrium through a needle inserted in the back. This method may give better definition of the left ventricle than a venous angiocardiogram, but it must carry a greater risk. Percutaneous puncture 
of the left ventricle and direct injection is described by Lehman (1959) who examined ten cases by this method without ill effect. However, this would appear to be a hazardous procedure especially if aneurysms are likely to be encountered. We believe the right atrial route to be the safest, and quite adequate for the purpose. It is a technique that could be used even if a leaking aneurysm is suspected.

\section{COMPLiCATIONS}

Traumatic aneurysms are liable to the same complications as those associated with coronary artery disease, but too few have been reported to allow any firm conclusions to be drawn as to the relative frequency. Rupture, an uncommon complication in non-traumatic cases, occurs comparatively frequently when trauma is the cause if one includes non-penetrating injuries (Groom 1897; Bright and Beck, 1935; Gunewardene, 1934; Hawkes, 1935; Pitts and Purvis, 1947; and others). Case 1 appears to be unique in surviving. Cardiac failure was observed at one time or another in almost all of the reported cases. It has been pointed out by Parkinson et al. (1938) that the hæmodynamic effects of an aneurysm resemble those of mitral and aortic regurgitation in that part of the left ventricular stroke volume does not pass into the systemic circulation and that diastolic filling is increased: hence, except when the aneurysm is small or thrombosed, the load on the heart is increased. Systemic embolism is a serious risk if the sac contains thrombus (Lyons and Perkins, 1958). Although patients with traumatic aneurysms may survive for long periods the risk of important complications appears to be sufficiently great to justify attempts at surgical cure.

\section{SuRgical TREATMENT}

The methods that were successful in dealing with each of our cases have been described in detail in the case reports. Exposure of an aneurysm involves the risk of catastrophic hæmorrhage. In Case 1 a large blood-loss was foreseen. It was fairly certain that the defect in the ventricle would not be larger than the original ventriculotomy, but a difficult approach was anticipated from the fact that the aneurysm had burst through the chest wall rather than into the pleural cavity. Preparations were therefore made for rapid blood replacement, but the loss was smaller than expected, and arterial transfusion was not required. Special precautions for dealing with hæmorrhage were not necessary in successfully treated chronic cases previously reported, nor in our Case 3, but in Case 2 with a comparatively large aneurysm of recent development, it was thought wise to be ready for any eventuality, and preparations were made for left ventricular bypass. The operation was completed without incident beyond the loss of only $400 \mathrm{ml}$. of blood.

The object in operating on any narrow-necked aneurysm is to close securely the defect in the heart wall. In Case 1 this was achieved simply by resuture with catgut. In the chronic stage of a false aneurysm it may still be possible to resuture the ventricle as was shown by Smith et al. (1957). Their method involved the introduction of a finger into the sac to control hæmorrhage during the repair. Bailey et al. (1958) suggest that there is little danger from emboli in traumatic aneurysms, but a finger in the sac must add to the risk of dislodging one, should clot be present.

Resection of a true aneurysm leads to some reduction in the capacity of the ventricle, for a tissue deficiency has to be overcome. This is minimized by the operation described by Likoff and Bailey (1955) and Bailey et al. (1958) as "ventriculoplasty". Fundamentally, it consists of subtotal resection of the sac and use of the tough fibromuscular neck of the aneurysm rather than adjacent muscle for the occluding sutures. This was the principle used in each of our chronic cases and, as Lyons and Perkins (1958) showed, the same method may be used for the cure of false aneurysms. In any operation on an aneurysm occlusion of the neck at the earliest possible moment, and before any manipulation of the sac, reduces the risks of dislodging thrombus and of rupturing the sac in the course of further dissection.

\section{SUMMARY}

Three left ventricular aneurysms that developed at the sites of small surgical incisions are reported. Of these, one was false and two were true. All were successfully repaired. 
The pathogenesis of traumatic aneurysms, true and false, is discussed. True aneurysms are ascribed to necrosis of a localized area of myocardium in the region of the cardiotomy. They are thus consequences of infarction and are in the same pathological category as the aneurysms that complicate coronary heart disease. False aneurysms are believed to be the result of partial dehiscence of the suture line from infection, or strangulation of the wound edges by the stitches.

The uncertainty of diagnosis by clinical and electrocardiographic means is mentioned. Radiological diagnosis is considered in detail with special reference to cineradiography and angiocardiography, and its value is stressed.

The complications of traumatic aneurysms are reviewed, and the principles of treatment are discussed.

We would like to thank Mr. W. P. Cleland and Dr. J. F. Goodwin for allowing us to study these patients and for encouragement and helpful criticism. Dr. David Lewes kindly referred Case 3.

\section{REFERENCES}

Bailey, C. P., Bolton, H. E., Nichols, H., and Gilman, R. A. (1958). J. thorac. Surg., 35, 37.

Barber, H. (1944). Quart J. Med., 13, 137.

Beck, C. S. (1944). Ann. Surg., 120, 34.

Bright, E. F., and Beck, C. S. (1935). Amer. Heart J., 10, 293.

Campbell, M., Deuchar, D. C., and Brock, Sir R. (1954). Brit med. J., 2, 111.

Derra, E., and Loogen, F. (1959). Dtsch. med. Wschr., 36, 1585.

Fatti, L. (1959). Personal communication.

Groom, W. (1897). Lancet, 1, 1202.

Gunewardene, H. O. (1934). Brit med. J., 2, 942.

Harken, D. E. (1946). Surg. Gynec. Obstet., 83, 117.

Hawkes, S. Z. (1935). Amer. J. Surg., 27, 503.

Lehman, J. S. (1959). Progress in Cardiovascular Disease, 2, 52.

Likoff, W., and Bailey, C. P. (1955). J. Amer. med. Asso., 158, 915.

Lyons, C., and Perkins, R. (1958). Ann. Surg., 147, 256.

McCord, M. C., and Blount, S. G. (1955) Circulation, 11, 754.

Myers, G. B., Klein, H. A., and Hiratzka, T. (1948). Amer. Heart J., 36, 836.

Parkinson, J., Bedford, D. E., and Thomson, W. A. R. (1938). Quart. J. Med., 7, 455.

Pitts, H. H., and Purvis, G. S. (1947). Canad. med. Ass. J., 57, 165.

Schlichter, J., Hellerstein, H. K., and Katz, L. N. (1954). Medicine, Baltimore, 33, 43.

Segal, N., Fatti, L., Adler, D., and Crawshaw, G. R. (1957). S. Afr. med. J., 31, 899.

Smith, R. C., Goldberg, H., and Bailey, C. P. (1957). Surgery, 42, 496.

Smithy, H. G., Boone, J. A., and Stallworth, J. M. (1950). Surg. Gynec. Obstet., 90, 175.

Thomas, C. G., and Ziffren, S. E. (1952). J. thorac. Surg., 24, 346.

Warren, W. D., Blanton, F. S., and Muller, W. H. (1957). Surgery, 42, 910.

Warren, W. D., Muller, W. H., East, M., and Sosa, O. (1958). Surg. Forum, 9, 257. 